\title{
Identification of a laccase gene involved in shell periostracal tanning of the clam Meretrix petechialis
}

\author{
Xin Yue ${ }^{1,2}$, Shujing Zhang ${ }^{1,3}$, Jiajia Yu ${ }^{1,3}$, Baozhong Liu ${ }^{1,2,3, *}$ \\ ${ }^{1}$ CAS Key Laboratory of Experimental Marine Biology, Institute of Oceanology, Center for Ocean Mega-Science, \\ Chinese Academy of Sciences, 7 Nanhai Road, Qingdao 266071, PR China \\ ${ }^{2}$ Laboratory for Marine Biology and Biotechnology, Qingdao National Laboratory for Marine Science and Technology, \\ 1 Wenhai Road, Qingdao 266000, PR China \\ ${ }^{3}$ University of Chinese Academy of Sciences, Beijing 100049, PR China
}

\begin{abstract}
Tanning is a complex extracellular process that is a mechanism for stabilizing proteinaceous extracellular structures. Phenoloxidases play important roles in cross-linking during tanning, and laccase (EC 1.10.3.2) is a member of the phenoloxidase enzyme class. In this study, we identified a laccase gene $(M p L a c)$ from the clam Meretrix petechialis and found that MpLaC might be involved in shell periostracal tanning of clams. Using whole-mount in situ hybridization, we found that MpLac mRNA in the larval clam was mainly expressed in the mantle edge. In the adult clam, our quantitative real-time PCR results showed that the mantle was also a tissue with a high MpLac expression level; in addition, by combining the results of fluorescence in situ hybridization, H\&E staining and transmission electron microscopy, we found that the inner epithelium of the outer fold of the mantle edge, which is involved in periostracum formation, was the exact region in which $M p L a c$ mRNA was expressed. Furthermore, knocking down the expression of MpLac by RNA interference (RNAi) bleached the new shell periostracum. All of our results suggest the involvement of $M p L a c$ in shell periostracal tanning of $M$. petechialis.
\end{abstract}

KEY WORDS: Meretrix petechialis $\cdot$ Laccase $\cdot$ Shell periostracum $\cdot$ Tanning

\section{INTRODUCTION}

Mollusks are generally covered with an exoskeleton (shell) consisting of calcium carbonate and an organic matrix (periostracum) (Lowenstam \& Weiner 1989, Marin \& Luquet 2004). The periostracum is mainly a quinone-tanned protein layer (Degens et al. 1967) and is among the mechanically strongest but chemically most inert structures in the animal kingdom (Waite 1983). The periostracum, which is located outside the shell surface, protects the calcified shell from corrosion and is also involved in shell calcification (Taylor \& Kennedy 1969). Periostracum formation originates in the periostracal groove (PG) of the mantle edge, and the proximal epithelium is

\footnotetext{
*Corresponding author: bzliu@qdio.ac.cn
}

involved in periostracum thickening (Bevelander \& Nakahara 1967, Kniprath 1972). First, the soluble precursor of the periostracum (i.e. periostracin) is secreted (Waite et al. 1979). Then, via a tanning process, periostracins are cross-linked and become rigid, forming the insoluble periostracum (Saleuddin \& Petit 1983).

Tanning (i.e. sclerotization and pigmentation) is a complex extracellular process first proposed $>70 \mathrm{yr}$ ago as a mechanism for stabilizing the proteinaceous extracellular structures found throughout the animal kingdom (Pryor 1940). Phenoloxidases play important roles in the cross-linking process during tanning (Andersen 2005, 2010, Checa 2000). In animals, there are 2 main types of phenoloxidases: tyrosinase

() The authors 2019. Open Access under Creative Commons by Attribution Licence. Use, distribution and reproduction are unrestricted. Authors and original publication must be credited. 
(EC.1.10.3.1) and laccase (EC.1.10.3.2). For laccase, 2 isoforms (laccase1 and laccase2) have been found in insects (Dittmer et al. 2004, Arakane et al. 2005). Laccase2 was reported to be indispensable for tanning of the cuticle (exoskeleton) in the insect Tribolium castaneum (Arakane et al. 2005). The involvement of laccase 2 in cuticle tanning has also been found in other insect species (Andersen 2005, Niu et al. 2008, Elias-Neto et al. 2013). In mollusks, laccase has been identified as an immune factor (Luna-Acosta et al. 2010, 2011, Le Bris et al. 2013, 2014), but little is known about its role in periostracum formation.

The Asiatic hard clam Meretrix petechialis is mainly distributed in the coastal areas of South and Southeast Asia and is an important commercial marine bivalve species (Tang et al. 2006). M. petechialis has a typical periostracum outside the calcified shell (Yue et al. 2015); thus, this species is an excellent model for investigation of shell periostracal tanning. In the present study, we identified a laccase-like gene from M. petechialis (MpLac) and investigated whether laccase is also involved in the tanning of the clam periostracum. We detected the expression patterns of MpLac in both larval and adult clams, and gained insights into the function of MpLac in shell periostracal tanning. Furthermore, in an RNA interference (RNAi) experiment, we observed changes in the shell periostracum when MpLac expression was knocked down. Our study will be helpful for understanding the molecular mechanisms underlying periostracum formation in clams.

\section{MATERIALS AND METHODS}

\subsection{Full-length cDNA cloning and sequence analysis}

After a basic local alignment search tool (BLAST) analysis of the expressed sequence tag (EST) sequences from the cDNA library of Meretrix petechialis that we had previously constructed (Yue et al. 2015), one EST sequence ( 400 bp) showed the highest similarity to the laccase gene. The full-length laccase cDNA of $M$. petechialis (MpLac) was then obtained by using the rapid amplification of cDNA ends (RACE). The $3^{\prime}$ and 5' RACE reactions were performed following the instructions of the SMART RACE CDNA amplification kit (Clontech). The PCR products were gel-purified and then individually cloned into the pMD19-T simple vector (TaKaRa). The recombinant plasmid was transformed into competent Escherichia coli cells, which were then cultured on Luria-Bertani
(LB) plates at $37^{\circ} \mathrm{C}$ overnight. Positive clones with the recombinant plasmid were identified by PCR. The recombinant plasmid was extracted from the mass culture of positive clones and then sequenced by the Sanger method. The entire assembled cDNA sequence was further verified by PCR amplification and sequencing.

The amino acid sequence of MpLac was deduced from the cDNA sequence. The signal peptide was predicted by both neural networks and hidden Markov models on the Signal IP 3.0 Server (Bendtsen et al. 2004). The isoelectric points (pI) and molecular weight of the deduced protein were determined using the 'Compute pI/MW' tool on the ExPASY Server (www.expasy.org/tools) (Gasteiger et al. 2005).

Phylogenetic analysis was done with amino acid sequences by the neighbour-joining (NJ) method with the program Mega 3.0. Besides MpLac, other laccases from different species (i.e. oyster Crassostrea gigas, freshwater snail Biomphalaria glabrata, amphioxus Branchiostoma belcheri, sponge Amphimedon queenslandica, silkworm Bombyx mori, moth Manduca sexta, green rice leafhopper Nephotettix cincticeps, beetle Tribolium castaneum) were subjected to the phylogenetic tree construction. The laccase from sponge $A$. queenslandica was set as an outgroup.

\subsection{Quantitative real-time PCR}

Using quantitative real-time PCR (qRT-PCR) with the specific primers lac-RT-F and lac-RT-R (Table 1), the relative mRNA expression of MpLac was compared among different clam tissues, including the adductor muscle, foot, hepatopancreas, haemolymph, mantle and gill. The different tissues of 5 clams were dissected separately and immediately stored at $-80^{\circ} \mathrm{C}$ for subsequent RNA extraction. In addition, qRT-PCR was also used to detect changes in the $M p L a c$ expression level in the mantle during the RNAi experiment. The samples used in the qRT-PCR are described in detail in Section 2.6.

Total RNA was extracted separately from each sample, and cDNA was synthesized as the qRT-PCR template. qRT-PCR was performed using a QuantiNova SYBR Green PCR Kit (Qiagen) with an ABI 7500 Fast Real-Time Thermal Cycler (ABI). The PCR parameters were $95^{\circ} \mathrm{C}$ for $30 \mathrm{~s}$, followed by 40 cycles of $95^{\circ} \mathrm{C}$ for $10 \mathrm{~s}$ and $60^{\circ} \mathrm{C}$ for $30 \mathrm{~s}$. Each $10 \mu \mathrm{l}$ volume of the PCR reaction mix contained $20 \mathrm{ng}$ of the template, $0.2 \mu \mathrm{M}$ of each primer and $5 \mu \mathrm{l}$ of the QuantiNova SYBR Green PCR Master Mix. Each reaction 
was performed in triplicate. $\beta$-actin was set as an internal reference to normalize expression levels among samples (Yue et al. 2011). The $2^{-\Delta \Delta C T}$ method was used to analyze the relative mRNA expression level of MpLac among samples (Livak \& Schmittgen 2001). Data were examined for homogeneity of variances by $F$-test and then analyzed by $t$-test using SPSS v.13.0 software. A difference was considered statistically significant if $\mathrm{p}<0.05$.

\subsection{Whole-mount in situ hybridization}

Clam larvae at 3 different developmental stages, including the D-veliger early stage, D-veliger late stage and postlarval stage, were separately collected and immediately fixed in $4 \%$ paraformaldehyde. To detect the locations of MpLac mRNA in the whole clam during development, whole-mount in situ hybridization (WMISH) was applied to the clam larvae. The detailed WMISH method is described in Yue et al. (2013). Briefly, a cDNA fragment of MpLac ( 600 bp) was amplified using the specific primers lac-ISH-F and lac-RT-R (Table 1) and then cloned into the pGEM-T-easy vector (Promega). A digoxigenin-labelled RNA probe with the MpLac antisense or sense sequence was synthesized following the general protocol of the DIG RNA labelling kit (Roche). The larvae were prehybridized in the hybridization buffer for $4 \mathrm{~h}$ at $65^{\circ} \mathrm{C}$. Then, the larvae were hybridized in the hybridization buffer with the antisense probe or the sense probe (negative control) for $12-14 \mathrm{~h}$ at $59^{\circ} \mathrm{C}$, followed by sequential washing. Blocking and antibody (i.e. alkaline phosphatase conjugated anti-digoxigenin antibody) incubation were subsequently performed. Finally, the larvae were stained with the colourimetric AP substrate (NBT/BCIP stock solution). The specific blue-violetcoloured precipitate indicated the location of $M p L a c$ mRNA. Images were captured with a digital camera mounted on a microscope (Nikon H600L).

\subsection{Fluorescence in situ hybridization}

The mantle edge zone was dissected from the adult clam and immediately fixed in $4 \%$ paraformaldehyde. Routine paraffin section preparation was applied to the mantle edge tissue. Sections with a $6 \mu \mathrm{m}$ thickness were cut and then transferred onto polyL-lysine-coated slides (Boster). To detect the mRNA expression of $M p L a c$ in the shell-forming tissue (i.e. mantle), these sections were analyzed with fluores- cence in situ hybridization (FISH) targeted to MpLac mRNA. The MpLac RNA probes used for FISH were the same as those used for WMISH. The FISH method was modified from that described in Yue et al. (2013). Briefly, the dewaxed sections on the slides were prehybridized in the hybridization buffer for $4 \mathrm{~h}$ at $65^{\circ} \mathrm{C}$ and then hybridized in the hybridization buffer with $1 \mathrm{ng} \mathrm{ul}^{-1}$ of the antisense or sense probe (negative control) for $12-14 \mathrm{~h}$ at $59^{\circ} \mathrm{C}$. After stringent washing and blocking, slides were incubated with anti-digoxigenin-fluorescein and Fab fragments (Roche) in blocking buffer for $2 \mathrm{~h}$ at $37^{\circ} \mathrm{C}$. Evans blue dye was used to contrast dye the non-antigen locations of the mantle edge. Unbound antibodies were removed by washing in 1× PBS. Finally, slides were mounted with ProLong Diamond Antifade Mountant (Invitrogen) and a coverslip and photographed with an LSM710 confocal laser scanning microscope (Zeiss).

\subsection{Hematoxylin and eosin staining and transmission electron microscopy}

To further determine the locations of MpLac mRNA in the mantle edge, hematoxylin and eosin (H\&E) staining and transmission electron microscopy (TEM) were performed. The microstructure of the mantle edge was revealed by H\&E staining, while the ultrastructure of the mantle edge was revealed by TEM. The detailed method of H\&E staining followed the descriptions in the 'H\&E staining protocol' on the website IHCWORLD (www.ihcworld.com/_protocols/ special_stains/h\&e_ellis.htm) (Martoja \& Martojapierson 1967). The TEM procedure followed the descriptions in Yue et al. (2010). To achieve accurate localization, semi-thin sections stained with $1 \%$ toluidine blue solution were prepared before cutting the ultrathin sections.

\subsection{RNAi}

RNAi was performed to detect the shell colour change when MpLac expression was knocked down. The cDNA fragment of MpLac was amplified using the T7 promoter-linked primer pair (lac-T7-F and lac-T7-R) (Table 1) and used as the PCR template to synthesize the MpLac double-stranded RNA (dsLac). The fragment of a pEGFP vector plasmid (Clontech) was amplified by EGFP-T7-F and EGFP-T7-R primers (Table 1) and used as the PCR template to synthesize the EGFP double-stranded RNA (dsEGFP). dsLac and dsEGFP were synthesized and purified 
Table 1. Primers used in this study

\begin{tabular}{|ll}
\hline Primer & Sequence $\left(5^{\prime}-3^{\prime}\right)$ \\
\hline lac-RT-F & CTA CCT GCT GGC GGT TAT GTC \\
lac-RT-R & CTC CTC CTC CGT GAA AGT CCT \\
lac-ISH-F & ATT TGC GAT GAA TCT AAG TGT GC \\
lac-T7-F & GCG TAA TAC GAC TCA CTA TAG GGA ACC AAT TAA CTA ACA CGC CTT TG \\
lac-T7-R & GCG TAA TAC GAC TCA CTA TAG GGG CTT ATC CCA TTC ATG TGA CCC \\
EGFP-T7-F & GCG TAA TAC GAC TCA CTA TAG GGA GCC ATA CCA CAT TTG TAG AGG \\
EGFP-T7-R & GCG TAA TAC GAC TCA CTA TAG GGC GCT TTC TTC CCT TCC TTT \\
\hline
\end{tabular}

with a TranscriptAid T7 High Yield Transcription Kit (Thermo) according to the manufacturer's instructions. The quantity and integrity of dsLac/dsEGFP were determined using a Nanodrop ND1000 spectrophotometer (Thermo) and agarose gel electrophoresis, respectively.

Clams with maroon shell colour (strain WM) (Yue et al. 2015) were acclimated in the laboratory for a week and then randomly divided into 3 groups, i.e. the dsLac-injected group, dsEGFP-injected group (negative control) and PBS-injected group (blank control). Based on different groups, $20 \mu \mathrm{l}$ of dsLac solution $\left(1.5 \mu \mathrm{g} \mathrm{l}^{-1}\right.$ in PBS), dsEGFP $\left(1.5 \mu \mathrm{g} \mathrm{\mu l^{-1 }}\right.$ in PBS) or PBS was injected into the mantle tissue of each clam. qRT-PCR was used to test the efficiency of the MpLac knockdown by detecting the change in the relative mRNA expression of MpLac during RNAi. Five clam mantles were collected from each group at 0, 24 and $48 \mathrm{~h}$ post-injection (hpi) for RNA extraction. Then the cDNA was synthesized and used as the qRT-PCR template. In order to monitor the changes in shell colour, the remaining 5 clams in each group were cultivated with injections of dsLac, dsEGFP or PBS once per week for $3 \mathrm{wk}$. All clams were photographed using a camera (SONY) before the 1st injection and after the 3rd injection.

\section{RESULTS}

\subsection{Characterization of the whole MpLac cDNA sequence}

As Fig. 1 shows, the complete cDNA sequence of $M p L a C$ with a poly-(A) tail is $2405 \mathrm{bp}$, including a 2130-bp open reading frame (ORF) encoding a polypeptide with 709 amino acid residues. Signal P prediction indicated that the $27 \mathrm{~N}$-terminal amino acids formed a signal peptide. The predicted mature protein was thus composed of 682 amino acids, with a theoretical mass of $76.35 \mathrm{kDa}$ and a pI of 5.67. The
cDNA and the deduced amino acid sequence have been deposited in GenBank under accession number MG845688. BLASTX searches against the NCBI database revealed that the deduced protein of MpLac shared the highest identity with a Crassostrea gigas laccase sequence.

The alignment result used for the phylogenetic tree construction is shown in Fig. S1 in the Supplement at www.int-res.com/articles/suppl/b028p055_supp.pdf. The phylogenetic tree (Fig. 2) showed that MpLaC has the closest relationship with the laccase of the freshwater snail Biomphalaria glabrata and the oyster C. gigas (XP_019920445.1, XP_019920446.1, EKC25244.1, EKC25250.1) but was distantly related to the laccases (1 and 2) of insect species. This result indicates that MpLac is systematically closer to the laccases from other mollusks than the insect laccases. Like insects, mollusk species may have multiple laccase isoforms, e.g. oyster C. gigas.

\subsection{Tissue distribution of MpLac mRNA in both larval and adult clams}

The distribution of MpLac mRNA in whole clam larvae at different developmental stages was detected by WMISH. At the early stage of D-veliger larvae $(24 \mathrm{~h}$ post-fertilization [hpf]), the time when the prodissoconch began to form, MpLac mRNA was detected along the mantle edge and occupied a large zone (Fig. 3a). With larval development, the mantle-edge zone with MpLac mRNA was reduced at the late D-veliger larvae stage (48 hpf) (Fig. 3b). At the postlarval stage $(10 \mathrm{~d}$ post-fertilization [dpf]), MpLac mRNA mainly appeared in the mantle edge and near the gill (Fig. 3c).

The tissue distribution of MpLac mRNA in adult clams was analysed by qRT-PCR. Results showed that MpLac mRNA was mainly expressed in the mantle and gill tissues and was expressed in other tissues at a significantly lower level ( $\mathrm{p}<0.05$ ) (Fig. 4). 
85 ATGGAAAGAACAACAATGGGCGCATATATTGGCCAATTGTATTTGATAACGATAATTGCCATCGTACCAGAAGCTATTTCTGACGGCCAC

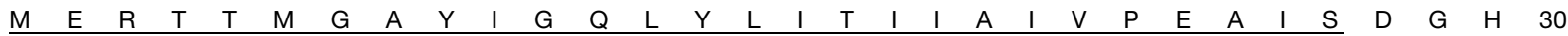

175 ACCAATCCAGGAACATGTGATATGGGCGCTTCAAAGTGTGACCTTTACCTTGATGTGAGCCTCTGGCTACCAATGCGACGTAACTATGGA

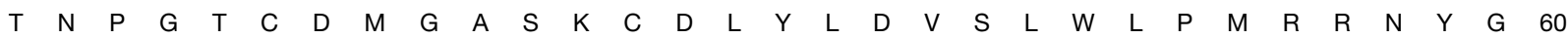
265 GAGAAAGTTTACATAAATGACACGGATGGTCGTTTATATGTACACGGTGATGAGTCGTACAATCAGATAGACCCAGATGATGTTGTCCTA

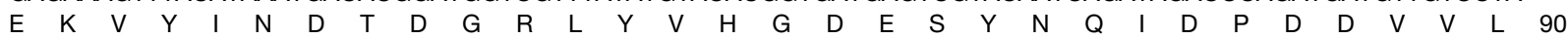

355 GCAGATGGATCGCCACGGGAACGAACACTGACACTTTTTAACAAGACAATGCCTGGCCCAACGTTAATTGTCTACGTTAATCAAGAGGTT

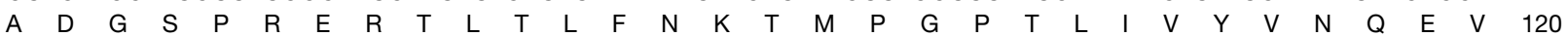
445 AAAGTTCACGTGAGGAATCAAATGTTAAGTGATGGAGTCACGGTCCATTTTCATGGTATTGAAATGCGAGGCACACCCTGGATGGACGGA

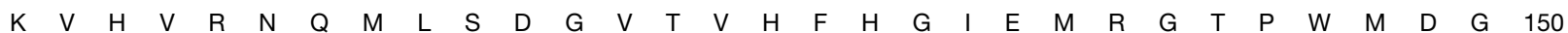
535 GCAGCGTTTGTCACCCAATGTCCGATTTTACCTGGACAAACGTTTACGTATAAGTTCACACCTAAGCGTAAGGGGACTTATTTTTATCAT

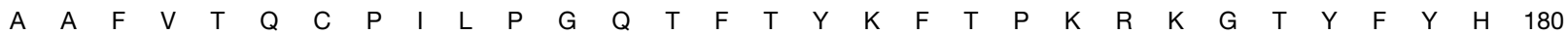
625 TCTCATACCGGCGCACAGATGAGTATGGGCTTGGTTGGGGCATTTATTGTCAAAGAGAGAAAATTAGATGAACTCGAGGAACATGTGATG

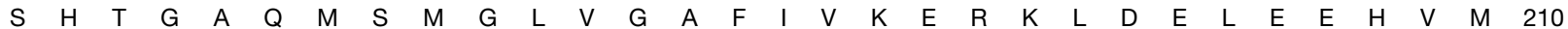
715 GTACTACAAGACTACAATAATGCACAGTCGTCCGATGAGTTATTTGCAAGTACAGGTTTACTAGGATTTATAAGTACAGAAGGTGAACCA $\begin{array}{llllllllllllllllllllllllllllllllllllllll}V & L & Q & D & Y & N & N & A & Q & S & S & D & E & L & F & A & S & T & G & L & L & G & F & I & S & T & E & G & E & P & 240\end{array}$ 805 TTTGCCTCAGAATTAAGAACTGACGGTTCGTACTCTGCTTCGATAAAAATAACATCGTCACTAATAAATGGCAAAGGCAGACTGTTCGAT $\begin{array}{lllllllllllllllllllllllllllllllllllll}F & A & S & E & L & R & T & D & G & S & Y & S & A & S & \text { I } & K & \text { I } & T & S & S & L & \text { I } & N & G & K & G & R & L & F & D & 270\end{array}$ 895 CCTCAGGGAAACCAATTAACTAACACGCCTTTGACTGTATTTCCTGTGAAGCAAGGCCAAGCTTATAGGTTCAGGGTAATCGGCGGGGCA $\begin{array}{llllllllllllllllllllllllllllllll}P & Q & G & N & Q & L & T & N & T & P & L & T & V & F & P & V & K & Q & G & Q & A & Y & R & F & R & V & I & G & G & A & 300\end{array}$ 985 TATTCCTTGCAACACAAAGTTTCTATAGACAATCATAAACTTAAACTAGTTGCAGCCGATGGGAACGACATTGATCCAATAATTGCCGAT

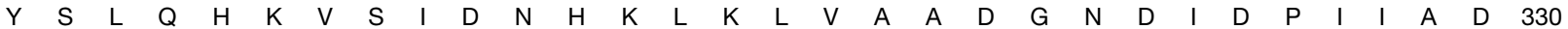
1075 TCTTTTATGATACATTCTGGAGAAAGATTTGATTTTATCATTGATGCTAATCAAACAGTTGATAATTATTGGATTAGAGCGGAAACATTG

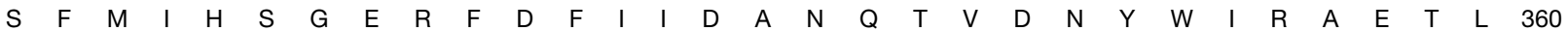
1165 CAGAAATATACTAACTATACAGGATTTGCAATCTTGCGATATGAGGGAGCTGAGAGTATTGATCCAACAAGTTCGGTTCAACAATGCTCA

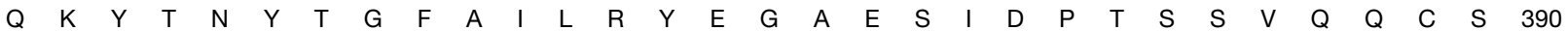
1255 ATGGAAAACATGTGTACAGTTGTCAACTGTCCTTTTGAAACGTATCCCAACTGGACCTGCATAACAACAGATCAAATTCACTCAACTACG $\begin{array}{llllllllllllllllllllllllllllllllll}M & E & N & M & C & T & V & V & N & C & P & F & E & T & Y & P & N & W & T & C & I & T & T & D & Q & I & H & S & T & T & 420\end{array}$ 1345 CCAGCTCCAACCCATAACTCTGGGAGTTTCAAAGAATTTTTCGTTAGCGTTGGATTTGCTCAGGCAGAGAACAATGTCTTTATGGGTCAC $\begin{array}{lllllllllllllllllllllllllllllllllllllllll}P & A & P & T & H & N & S & G & S & F & K & E & F & F & V & S & V & G & F & A & Q & A & E & N & N & V & F & M & G & H & 450\end{array}$ 1435 ATGAATGGGATAAGCCTTAAGTTACCAAGTGTTTCCGCATTAACGCAACCAAAAGAGGTGACTGATATTTGCGATGAATCTAAGTGTGCG $\begin{array}{llllllllllllllllllllllllllllllll}M & N & G & I & S & L & K & L & P & S & V & S & A & L & T & Q & P & K & E & V & T & D & I & C & D & E & S & K & C & A & 480\end{array}$ 1525 GCGGGTAAATTTTGCAGATGTTATCGTTCTCTTGAAGTGAATTTCGGAGATGTCGTACAGATAAACTTCTTCAGTGCAGGAAAATATAAC $\begin{array}{llllllllllllllllllllllllllllllllllllll}A & G & K & F & C & R & C & Y & R & S & L & E & V & N & F & G & D & V & V & Q & I & N & F & F & S & A & G & K & Y & N & 510\end{array}$ 1615 CTTGCCAGCCACCCTATACACATACATGGATACTCGTTTCACGTTTTAAAAGTTGGTTATCGGCAGGAAAATTCCAGCGTTGTAAATGCT $\begin{array}{lllllllllllllllllllllllllllllllll}L & A & S & H & P & \text { I } & H & \text { I } & H & G & Y & S & F & H & V & L & K & V & G & Y & R & Q & E & N & S & S & V & V & N & A & 540\end{array}$ 1705 CAATTCCTTAATCAATATATCAGTTGTCCAAACGAATTTTGTTACCGTAATTTGGGATGGGCTAATCAAAGCTGGAAAGGAGGAAACATT

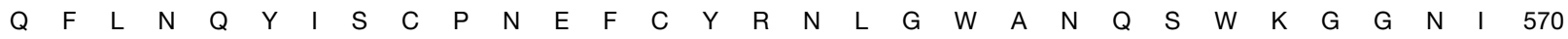
1795 CCCGGGATTGATTTAGATCGGGCACCGCGTAAGGATACAGTTACCCTACCTGCTGGCGGTTATGTCGTTGTAAGATTTGTTGCAGACAAT $\begin{array}{llllllllllllllllllllllllllllllllllllll}P & G & I & D & L & D & R & A & P & R & K & D & T & V & T & L & P & A & G & G & Y & V & V & V & R & F & V & A & D & N & 600\end{array}$ 1885 CCAGGTCTCTGGTACATCCATTGTCACCAAGAATACCATGCTCAAAAGGGTTTAGGATTACTACTTAATGATTCATTCTCAGCAATCCCT

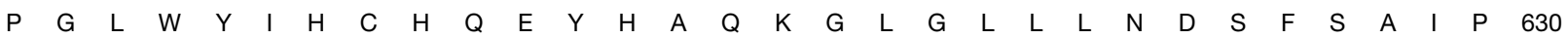
1975 GCCCCACCCGAGGGATTCCTGAATGTAGAAGCTTCCGCCTCCAGACTGGCTGACTTCAGACAAACGGGAATCATTGCCCAAAGACGAT

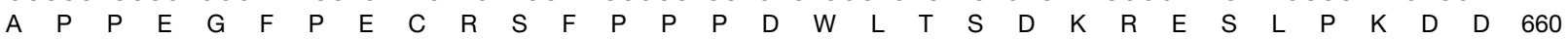
2065 GAAAATACAACAGTTGCACGTACAGACGGAAGGACTTTCACGGAGGAGGAGTTCTGGGGAATGTTTGGAGCGCTTCTGTTCGTGATTTTA $\begin{array}{lllllllllllllllllllllllllllllll}N & T & T & V & A & R & T & D & G & R & T & F & T & E & E & E & F & W & G & M & F & G & A & L & L & F & V & I & L & 690\end{array}$ 2155 TTACAGTTCATCATTTCTGTGTGGTGCTTAAGAAAAACCAAATCTTCCGTATCGTTCTAGaacagacaaaagaagaaaaaagtacacttt $L \quad Q \quad F \quad I \quad I \quad S \quad V \quad W \quad C \quad L \quad R \quad K \quad T \quad K \quad S \quad S \quad V \quad S \quad F \quad$ *

2245 tggattaaataacttagtattttagttctcattcctctgtatgtattcacaaactgctgtatttataaaaactgtaactcgcttt

2335 ataagatttttatgtaacctcgttgagatgaatacaattaaagacaaaaaaaaaaaaaaaaa

Fig. 1. Nucleotide sequence (upper) and deduced amino acid sequence (single letter code, below) of the full-length $M p L a c$ cDNA. Nucleotide and amino acid numbers are shown on the left and right, respectively. Underlined amino acids are the predicted signal peptide. *: stop codon

\subsection{MpLac mRNA localization in the mantle edge of adult clams}

Based on the results of H\&E staining (Fig. 5a), the mantle edge of $M$. petechialis was divided into 3 folds: the inner fold (IF), middle fold (MF) and outer fold (OF). The PG was between the MF and OF; the periostracum originated from the PG and adjoined the inner epithelium of the $\mathrm{OF}_{\text {; }}$ the periostracum was thin near the PG and intensified gradually with distance from the PG. These results imply that the inner epithelium of the OF plays an important role in periostracum formation. TEM results also showed that large amounts of electron-opaque granules were secreted from the inner epithelium of the OF to the electron-dense periostracum (Fig. 5b), further implying that the inner epithelium of the OF is involved in periostracal secretion. 


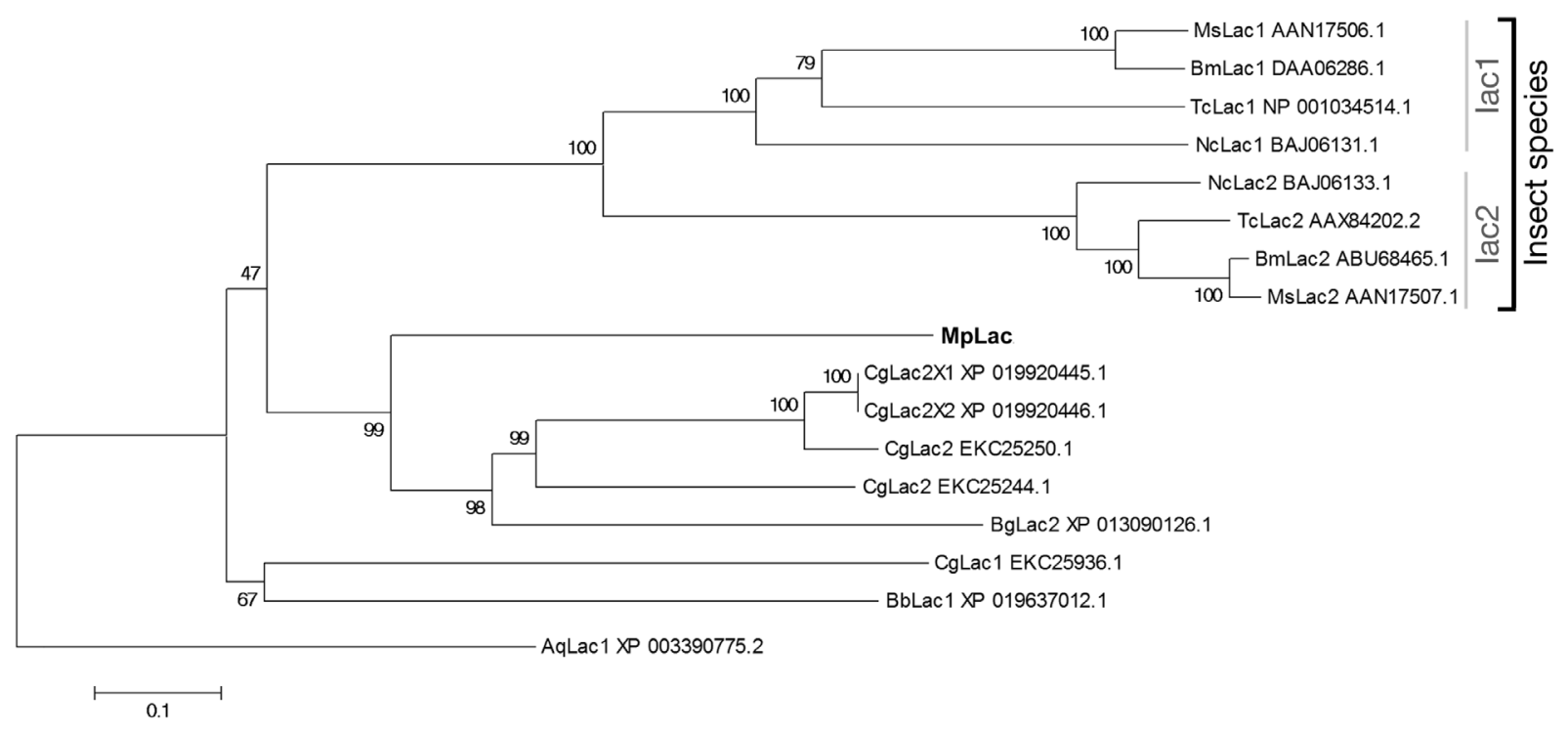

Fig. 2. Phylogenetic tree depicting the relationship between MpLac (bold) and laccases of other species. The sequences applied to the phylogenetic tree construction were as follows: Crassostrea gigas (Cg), laccase-2 isoform X1, laccase-2 isoform X2, laccase-2, laccase-2, laccase-1; Biomphalaria glabrata (Bg), laccase-2; Branchiostoma belcheri (Bb), laccase-1; Amphimedon queenslandica (Aq), laccase-1; Bombyx mori (Bm), laccase-1, laccase-2; Manduca sexta (Ms), laccase-1, laccase-2; Nephotettix cincticeps (Nc), laccase-1, laccase-2; Tribolium castaneum (Tc), laccase-1, and laccase-2. The laccase from sponge A. queenslandica was set as an outgroup. The tree was constructed using the neighbor joining method; bootstrap confidence was calculated from 1000 replications. The scale bar represents 0.1 amino acid substitutions per site

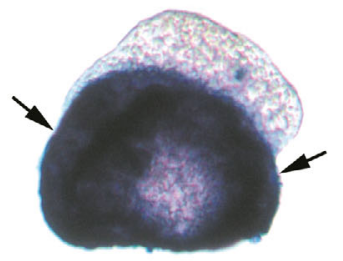

a

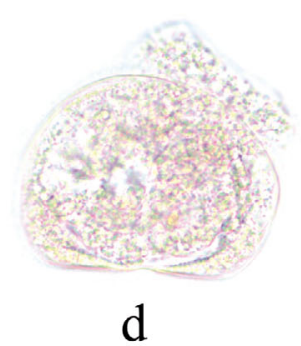

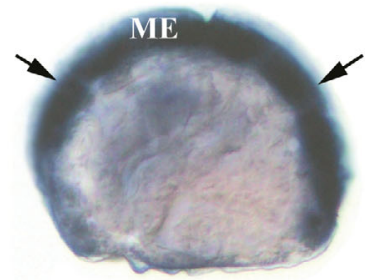

b

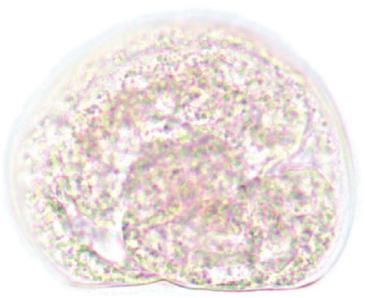

e

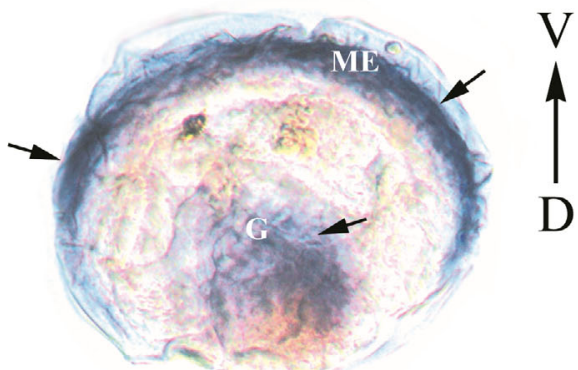

C

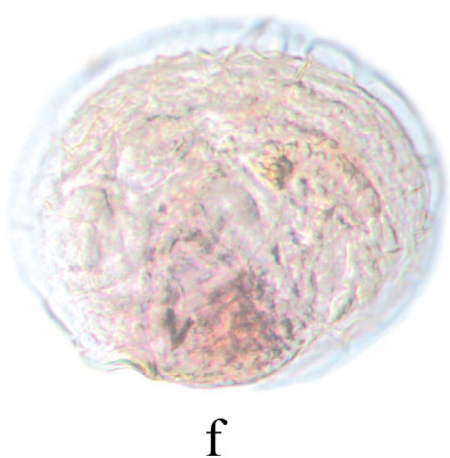

Fig. 3. MpLac mRNA expression at different larval stages of Meretrix petechialis revealed by whole-mount in situ hybridization, showing larvae hybridized with $(\mathrm{a}-\mathrm{c})$ the antisense probe and $(\mathrm{d}-\mathrm{f})$ the sense probe (negative control). Lateral view of the $(\mathrm{a}, \mathrm{d})$ early D-veliger larva; $(\mathrm{b}, \mathrm{e})$ late D-veliger larva; and $(\mathrm{c}, \mathrm{f})$ postlarvae. Arrowheads: positive staining (blue-violet colour) of MpLac mRNA. ME: mantle edge; G: gill; V: venter; D: dorsum 


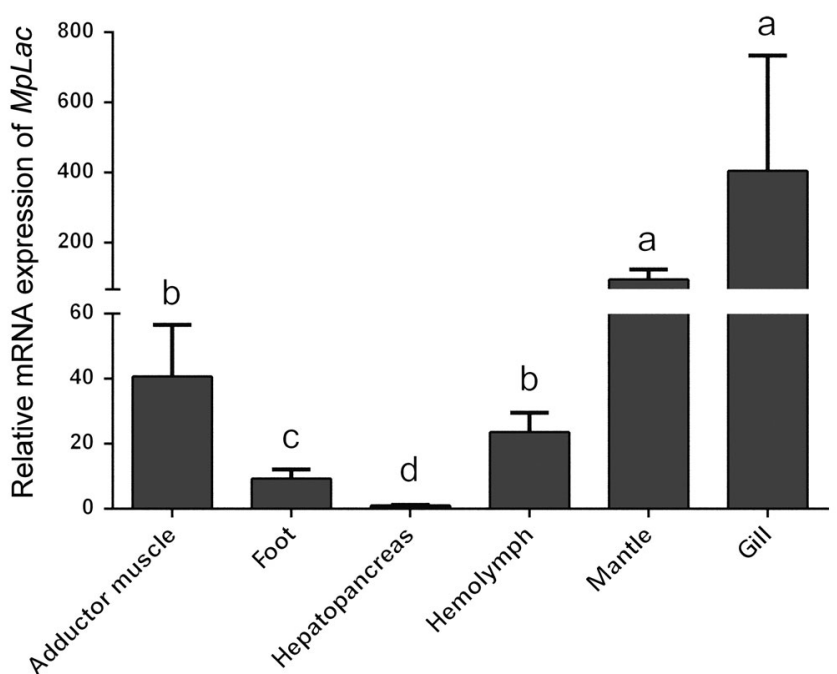

Fig. 4. Relative mRNA expression of MpLac in various tissues of adult clams. Error bars: SD. Columns with different letters are significantly different from each other $(\mathrm{p}<0.05)$

Since the periostracum formed in the mantle edge of the clam, we detected MpLac expression in the mantle edge by FISH to identify the function of $M p L a C$ in periostracum formation. FISH results showed that the MpLac mRNA was located in the mantle-edge epithelium and was mainly limited to the inner epithelium of the $\mathrm{OF}$, which was adjacent to the periostracum (Fig. 6a).

\subsection{Change in periostracum colour after knocking down MpLac expression}

To test whether the shell colour (i.e. the periostracum colour) of clams is related to MpLac expression level, an RNAi experiment was performed. The qRT-PCR results showed that at $24 \mathrm{hpi}, M p L a c$ expression level was significantly decreased in the dsLac-injected group compared to the PBS-injected group (blank control) and dsEGFP-injected group (negative control) $(\mathrm{p}<0.05)$ (Fig. 7). At $48 \mathrm{hpi}$, $M p L a c$ expression level was significantly lower in the dsLac-injected group than the dsEGFP-injected group $(p<0.05)$. Compared to the PBS-injected group, the mean of MpLac expression level was $25 \%$ lower in the dsLac-injected group. The nonsignificant difference in $M p L a c$ expression level between the dsLac-injected and PBS-injected group was because there was a large standard deviation root in the huge expression difference among the PBS-injected clams (Fig. 7). Thus, we think the knockdown may be still effective at $48 \mathrm{hpi}$. Changes in the shell colour were checked for the clams in all 3 groups. Our results showed that in the dsLac-injected clams, the colour of the new shell was bleached (Figs. 8 \& S2). No such changes were detected in the dsEGFP- and PBS-injected clams (Fig. 8).
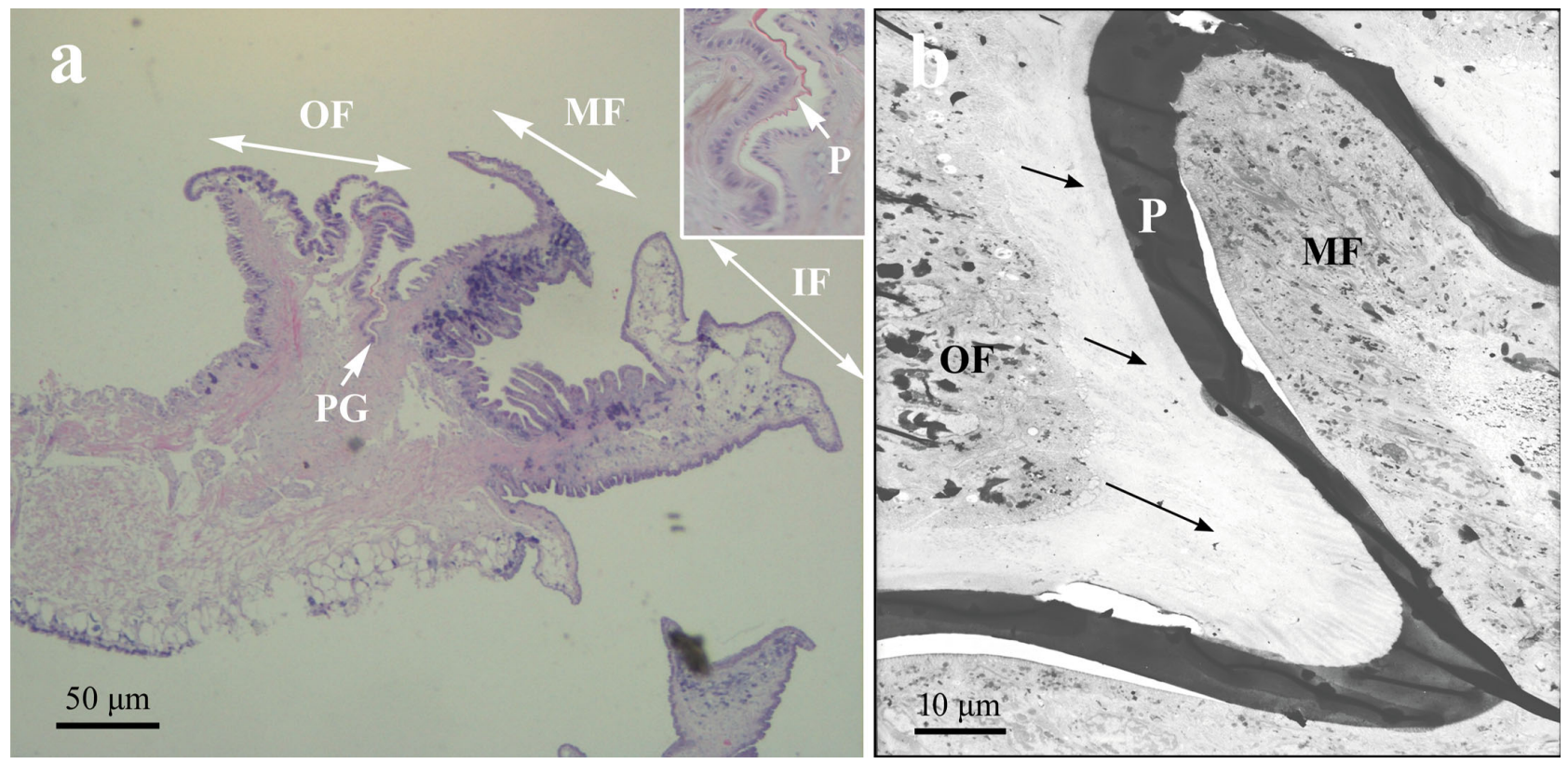

Fig. 5. (a) Microstructure of the mantle edge of the adult clam revealed by H\&E staining and (b) ultrastructure revealed by transmission electron microscopy. The magnified image of the periostracal groove (PG) region is shown at the top-right corner of image (a). OF: outer fold; MF: middle fold; IF: inner fold; P: periostracum. Black arrows: massive granules being secreted from the inner epithelium of the OF to the $P$ 

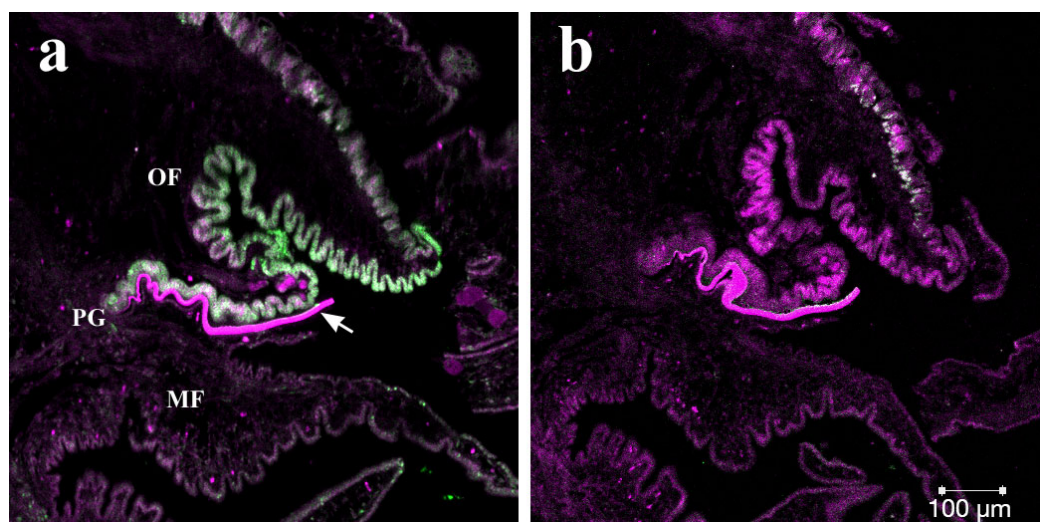

Fig. 6. MpLac mRNA localization in the mantle edge of the adult clam revealed by fluorescence in situ hybridization, showing slides hybridized with (a) the antisense probe and (b) the sense probe (negative control). Green fluorescence signals indicate $M p L a c$ mRNA. OF: outer fold; MF: middle fold; PG: periostracal groove. White arrow indicates the periostracum

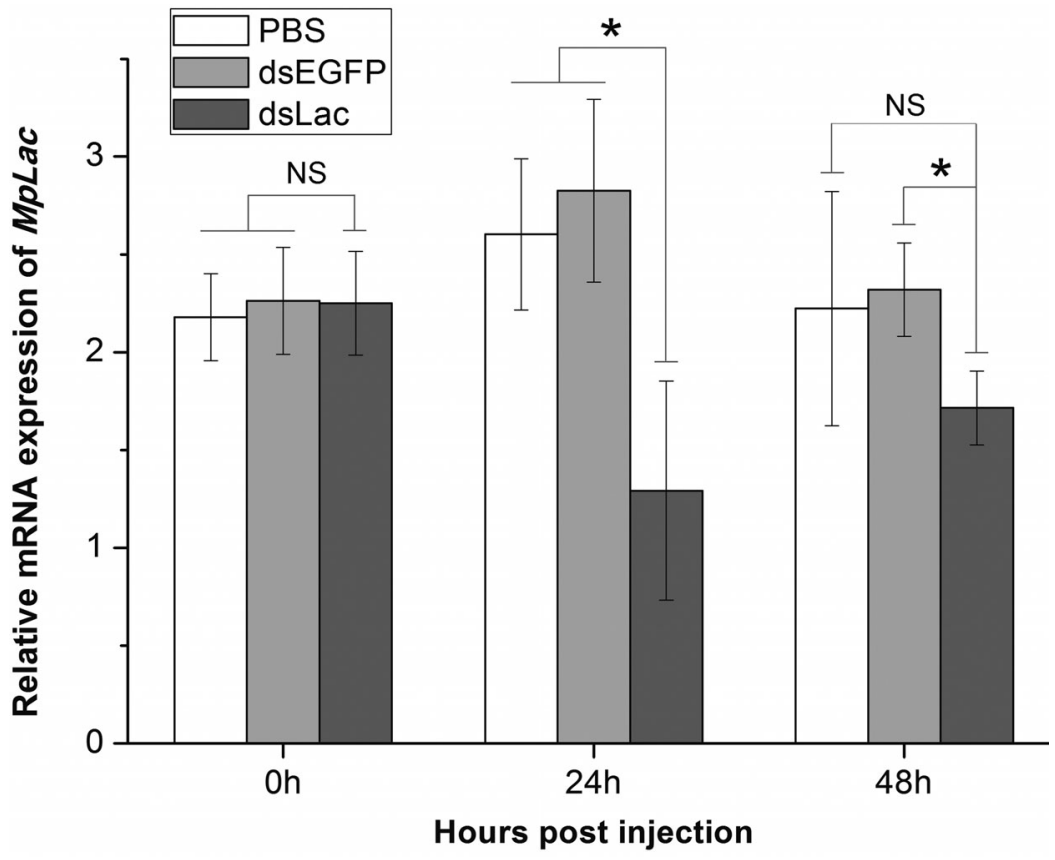

Fig. 7. Relative mRNA expression of $M p L a c$ in the mantle of clams injected with dsLac/dsEGFP/PBS (see Section 2.6). Error bars: SD. $\left({ }^{*}\right)$ represent a significant difference $(\mathrm{p}<0.05)$ between the dsLac-injected group and any of the control groups (dsEGFP/PBS-injected groups). NS: no significant difference

\section{DISCUSSION}

Laccases comprise a group of multi-copper enzymes. In many insects, laccase has been reported to be involved in the tanning of larval, pupal and adult cuticles (Andersen 2005, Arakane et al. 2005, Niu et al. 2008, Elias-Neto et al. 2013). In this study, we identified a laccase gene from the clam Meretrix petechialis and investigated whether laccase was also involved in the tanning of the clam periostracum.

M. petechialis has a typical periostracum outside the calcified shell; the colour of this periostracum determines the colour of the shell (Yue et al. 2015). Periostracum synthesis starts at the early stages of development, and the periostracum grows continuously over the entire life of the bivalve. The larval shell of the bivalve undergoes 3 stages during ontogenesis, namely prodissoconch-I, prodissoconch-II and dissoconch, all of which contain the periostracum structure (Marin \& Luquet 2004). The prodissoconch-I and prodissoconch-II form in the course of D-veliger larval development, and the dissoconch appears after metamorphosis of the veliger larva to the juvenile larva (Che et al. 2001). In this study, with the WMISH method, MpLac expression was detected in 3 developmental stages of clam larvae, i.e. the early stage of D-veliger larvae, the late stage of D-veliger larvae and the postlarval stage, which separately secreted the prodissoconch-I, prodissoconch-II and dissoconch shells. The WMISH results (Fig. 3) showed that MpLac mRNA was detected in all 3 larval stages and mainly appeared along the mantle edge. The zone of the mantle edge with MpLac mRNA is larger in the early D-veliger larvae than in the late D-veliger larvae and postlarvae; this may be because the shell field spreads with the fast lateral extension of the periostracum during the growth of the prodissoconch-I shell (Marin \& Luquet 2004) or due to the undifferentiation of the mantle edge at the early D-veliger larval stage (Arandaburgos et al. 2014). Shell growth in bivalves is related to the activities of mantle epithelial cells (Jolly et al. 2004). The periostracum, as a part of the mollusk shell, is secreted by the mantle (Addadi \& Weiner 1985). Our results, that $M p L a c$ transcripts were mainly expressed in the mantle edge of the larval clam, suggest that MpLac is likely involved in the formation of the larval clam periostracum. 


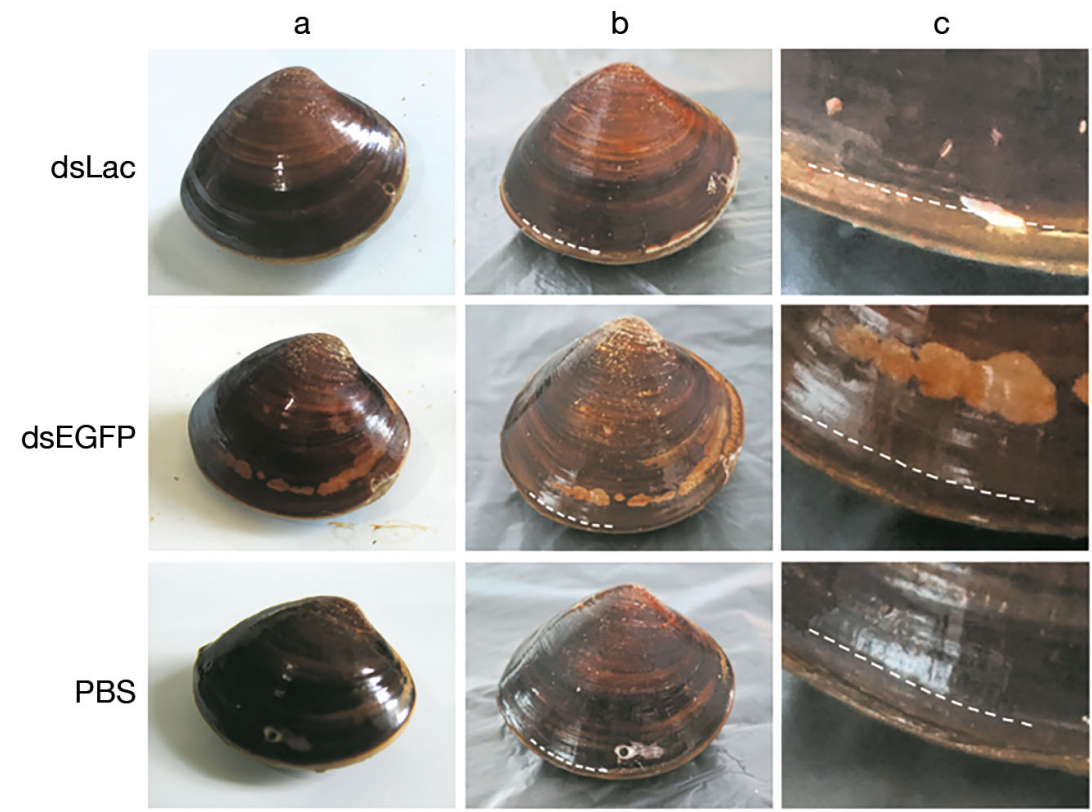

Fig. 8. Changes in periostracum colour among clams injected with dsLac/ dsEGFP/PBS (see Section 2.6). (a) Clam images before the first injection; (b) clam images after the last injection; (c) magnified shell-margin images of the clams after the last injection. Dashed line: boundary between the old and new shells

In the adult clam, similar to in the larval clam, the mantle was also a tissue with a high expression level of MpLac (Fig. 4), further implying the involvement of $M p L a c$ in periostracum formation. Results of H\&E staining and TEM (Fig. 5) showed that the periostracum of the adult clam originated in the PG of the mantle edge, and the inner epithelium of the OF was involved in periostracum thickening. Our results were consistent with the results in other bivalve species (Bevelander \& Nakahara 1967, Kniprath 1972). Notably, the FISH results (Fig. 6) showed that MpLac transcripts were not only located in the epithelium of the mantle edge but were also mainly limited to the region of the inner epithelium of the OF. These results imply that MpLac is involved in the periostracum formation of the adult clam. The expression locations of MpLac in both larval and adult clams suggest a close relationship between MpLac and the periostracum.

Additionally, an RNAi experiment was performed to verify the role MpLac plays in periostracum tanning of the clam $M$. petechialis. RNAi is a widely used method to silence gene expression in mollusks (Suzuki et al. 2009). For instance, RNAi was used to suppress the expressions of some shell-related genes in the pearl oyster, and the influence of RNAi on shell formation was observed (Fang et al. 2011, Jiao et al. 2012); RNAi was also used to identify the down- stream genes of the signalling pathway in scallops and pearl oysters (Wang et al. 2011, Oyanedel et al. 2016, Zhang et al. 2017). A previous study showed that RNAi of laccase-2 in the insect Tribolium castaneum made the cuticle bleach; thus, laccase is the phenoloxidase gene required for beetle cuticle tanning (Arakane et al. 2005). In this study, an RNAi experiment was performed to detect the change in shell colour (i.e. the periostracum colour) of clams after the expression of MpLac was knocked down. Strain WM, developed by our lab and displaying an overall maroon shell-colour (Yue et al. 2015), was used in the RNAi experiment. During the RNAi experiment, we found that the new shell colour of the clam was bleached after MpLac expression was knocked down (Figs. 8 \& S2). Even though the MpLac expression began to increase again at $48 \mathrm{hpi}$, an obvious colour bleaching was observed in the dsLac-injected clams, which may be due to the additive effect of repeated RNAi. The results of RNAi further suggested that $M p L a c$ was a phenoloxidase gene required for the periostracal tanning of clams.

The complete cDNA sequence of MpLac we obtained was $2405 \mathrm{bp}$, including a 2130-bp ORF encoding a polypeptide with 709 amino acid residues (Fig. 1). The results of our phylogenetic analysis (Fig. 2) showed that MpLac is systematically closer to the laccases from other mollusks than the insect laccases. Asano et al. (2014) also indicated that mollusk laccases, which were devoid of the cysteine-rich domain, clustered together and were isolated from the cluster of insect laccases, implying that mollusk laccases represent a new sub-family of laccases. Our study identified a laccase gene from the clam $M$. petechialis and demonstrates the relationship between the laccase (MpLac) and shell periostracal tanning for the first time.

In conclusion, a laccase-like gene (MpLac) was identified in the clam M. petechialis, and the MpLac transcripts were mainly expressed in the mantle edge of both larval and adult clams. Furthermore, $M p L a c$ transcripts were mainly limited to the inner epithelium region of the OF of the mantle edge, which is responsible for periostracum thickening. In addition, knocking down MpLac expression bleached the new shell periostracum. All of our 
results indicate the involvement of MpLac in the shell periostracal tanning of $M$. petechialis. This study will be helpful for understanding the molecular mechanisms underlying periostracum formation in clams.

Acknowledgements. This work was funded by China Agriculture Research System (CARS-49), the Youth Foundation Project (YQ2018NO07) from Qingdao National Laboratory for Marine Science and Technology, and National Natural Science Foundation of China (NSFC 31772845).

\section{LITERATURE CITED}

Addadi L, Weiner S (1985) Interactions between acidic proteins and crystals: stereochemical requirements in biomineralization. Proc Natl Acad Sci USA 82: 4110-4114

Andersen SO (2005) Cuticular sclerotization and tanning. In: Gilvert LI, Iatrou K, Gill SS (eds) Comprehensive molecular insect science, Vol 4: biochemistry and molecular biology. Elsevier, Oxford, p 145-170

Andersen SO (2010) Insect cuticular sclerotization: a review. Insect Biochem Mol Biol 40:166-178

Arakane Y, Muthukrishnan S, Beeman RW, Kanost MR, Kramer KJ (2005) Laccase 2 is the phenoloxidase gene required for beetle cuticle tanning. Proc Natl Acad Sci USA 102:11337-11342

*Arandaburgos JA, Costa FD, Nóvoa S, Ojea J, Martínezpatiño D (2014) Embryonic and larval development of Ruditapes decussatus (Bivalvia: Veneridae): a study of the shell differentiation process. J Molluscan Stud 80:8-16

Asano T, Taoka M, Yamauchi Y, Everroad RC and others (2014) Re-examination of a $\alpha$-chymotrypsin-solubilized laccase in the pupal cuticle of the silkworm, Bombyx mori: insights into the regulation system for laccase activation during the ecdysis process. Insect Biochem Mol Biol 55:61-69

Bendtsen JD, Nielsen H, von Heijne G, Brunak S (2004) Improved prediction of signal peptides: SignalP 3.0. J Mol Biol 340:783-795

Bevelander G, Nakahara H (1967) An electron microscope study of the formation of the periostracum of Macrocallista maculata. Calcif Tissue Res 1:55-67

Che LM, Golubic S, Campion-Alsumard TL, Payri C (2001) Developmental aspects of biomineralisation in the Polynesian pearl oyster Pinctada margaritifera var. cumingii. Oceanol Acta 24:37-49

Checa A (2000) A new model for periostracum and shell formation in Unionidae (Bivalvia, Mollusca). Tissue Cell 32:405-416

Wegens ET, Spencer DW, Parker RH (1967) Paleobiochemistry of molluscan shell proteins. Comp Biochem Physiol 20:553-579

Dittmer NT, Suderman RJ, Jiang H, Zhu YC and others (2004) Characterization of cDNAs encoding putative laccase-like multicopper oxidases and developmental expression in the tobacco hornworm, Manduca sexta, and the malaria mosquito, Anopheles gambiae. Insect Biochem Mol Biol 34:29-41

Elias-Neto M, Soares MPM, Bitondi MMG (2013) Expression profile of a Laccase 2 encoding gene during the metamorphic molt in Apis mellifera (Hymenoptera, Apidae). Rev Bras Entomol 57:213-216

F Fang D, Xu G, Hu Y, Pan C and others (2011) Identification of genes directly involved in shell formation and their functions in pearl oyster, Pinctada fucata. PLOS ONE 6:e21860

Gasteiger E, Hoogland C, Gattiker A, Duvaud S and others (2005) Protein identification and analysis tools on the ExPASy server. In: Walker JM (ed) The proteomics protocols handbook. Humana Press, Totowa, NJ, p 571-607

* Jiao Y, Wang H, Du X, Zhao X and others (2012) Dermatopontin, a shell matrix protein gene from pearl oyster Pinctada martensii, participates in nacre formation. Biochem Biophys Res Commun 425:679-683

Jolly C, Berland S, Milet C, Borzeix S, Lopez E, Doumenc D (2004) Zona localization of shell matrix proteins in mantle of haliotis tuberculata (Mollusca, Gastropoda). Mar Biotechnol (NY) 6:541-551

Kniprath E (1972) Formation and structure of the periostracum in Lymnaea stagnalis. Calcif Tissue Res 9:260-271

Le Bris C, Paillard C, Stiger-Pouvreau V, Guérard F (2013) Laccase-like activity in the hemolymph of Venerupis philippinarum: characterization and kinetic properties. Fish Shellfish Immunol 35:1804-1812

Le Bris C, Lepretre M, Paillard C, Guérard F (2014) Characterization of a laccase-like activity in the hemolymph of the abalone Haliotis tuberculata. Aquaculture 424425:194-200

* Livak KJ, Schmittgen TD (2001) Analysis of relative gene expression data using real-time quantitative PCR and the $2^{-\Delta \Delta \mathrm{CT}}$ Method. Methods 25:402-408

Lowenstam HA, Weiner S (1989) On biomineralization. Oxford University Press, New York, NY

* Luna-Acosta A, Rosenfeld E, Amari M, Fruitier-Arnaudin I and others (2010) First evidence of laccase activity in the Pacific oyster Crassostrea gigas. Fish Shellfish Immunol 28:719-726

* Luna-Acosta A, Saulnier D, Pommier M, Haffner P and others (2011) First evidence of a potential antibacterial activity involving a laccase-type enzyme of the phenoloxidase system in Pacific oyster Crassostrea gigas haemocytes. Fish Shellfish Immunol 31:795-800

* Marin F, Luquet G (2004) Molluscan shell proteins. C R Palevol 3:469-492

Martoja R, Martojapierson M (1967) Initiation aux techniques de l'histologie animale. Masson et Cie, Paris

Niu BL, Shen WF, Liu Y, Weng HB and others (2008) Cloning and RNAi-mediated functional characterization of MaLac2 of the pine sawyer, Monochamus alternatus. Insect Mol Biol 17:303-312

* Oyanedel D, Gonzalez R, Flores-Herrera P, Brokordt K and others (2016) Molecular characterization of an inhibitor of NF-B in the scallop Argopecten purpuratus: first insights into its role on antimicrobial peptide regulation in a mollusk. Fish Shellfish Immunol 52:85-93

Pryor MGM (1940) On the hardening of the cuticle of insects. Proc Natl Acad Sci USA 128:393-407

Saleuddin ASM, Petit HP (1983) The mode of formation and the structure of the periostracum. In: Saleuddin ASM, Wilbur KM (eds) The Mollusca, Vol 4: physiology. Academic Press, New York, NY, p 199-233

* Suzuki M, Saruwatari K, Kogure T, Yamamoto Y and others (2009) An acidic matrix protein, Pif, is a key macromolecule for nacre formation. Science 325:1388-1390 
Tang B, Liu B, Wang G, Zhang T, Xiang J (2006) Effects of various algal diets and starvation on larval growth and survival of Meretrix meretrix. Aquaculture 254:526-533

Taylor JD, Kennedy WJ (1969) The influence of the periostracum on the shell structure of bivalve molluscs. Calcif Tissue Res 3:274-283

Waite JH (1983) Quinone-tanned scleroproteins. In: Saleuddin ASM, Wilbur KM (eds) The Mollusca, Vol 4: physiology. Academic Press, New York, NY, p 467-504

Waite JH, Saleuddin ASM, Andersen SO (1979) Periostracin-a soluble precursor of sclerotized periostracum in Mytilus edulis L. J Comp Physiol 130:301-307

Wang M, Yang J, Zhou Z, Qiu L and others (2011) A primitive Toll-like receptor signaling pathway in mollusk Zhikong scallop Chlamys farreri. Dev Comp Immunol 35: $511-520$

Yue X, Liu B, Xiang J, Jia J (2010) Identification and characterization of the pathogenic effect of a Vibrio parahaemo-

Editorial responsibility: Nikolaos Schizas,

Mayagüez, Puerto Rico, USA lyticus-related bacterium isolated from clam Meretrix meretrix with mass mortality. J Invertebr Pathol 103: $109-115$

* Yue X, Liu B, Xue Q (2011) An i-type lysozyme from the Asiatic hard clam Meretrix meretrix potentially functioning in host immunity. Fish Shellfish Immunol 30:550-558

* Yue X, Huan P, Xiao G, Liu B (2013) Expression patterns of an i-type lysozyme in the clam Meretrix meretrix along with larval development. Dev Comp Immunol 41: 27-32

Yue X, Nie Q, Xiao G, Liu B (2015) Transcriptome analysis of shell color-related genes in the clam Meretrix meretrix. Mar Biotechnol (NY) 17:364-374

Khang H, Zhao M, Yi X, Ou Z and others (2017) Characterization of the distal-less homologue gene, PfDlx, involved in regulating the expression of $P$ if in the pearl oyster, Pinctada fucata. Comp Biochem Physiol B Biochem Mol Biol 212:51-58

Submitted: August 21, 2018; Accepted: April 1, 2019

Proofs received from author(s): June 3, 2019 